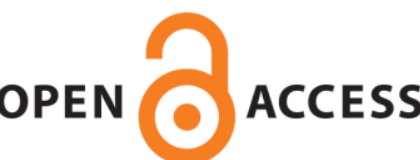

UWS Academic Portal

\title{
Dipole response of the odd-proton nucleus 205TI up to the neutron-separation energy
}

Benouaret, Nadia; Beller, J.; Pai, H.; Pietralla, N.; Ponomarev, V. Yu.; Romig, C.;

Schnorrenberger, L.; Zweidinger, M.; Scheck, Marcus; Isaak, J.; Savran, D.; Sonnabend, K.; Raut, R.; Rusev, G.; Tonchev, A. P.; Tornow, W.; Weller, H. R.; Kelley, J. H.

Published in:

Journal of Physics G: Nuclear and Particle Physics

DOI:

10.1088/0954-3899/43/11/115101

Published: 17/10/2016

Document Version

Peer reviewed version

Link to publication on the UWS Academic Portal

Citation for published version (APA):

Benouaret, N., Beller, J., Pai, H., Pietralla, N., Ponomarev, V. Y., Romig, C., Schnorrenberger, L., Zweidinger, M., Scheck, M., Isaak, J., Savran, D., Sonnabend, K., Raut, R., Rusev, G., Tonchev, A. P., Tornow, W., Weller, H. R., \& Kelley, J. H. (2016). Dipole response of the odd-proton nucleus 205TI up to the neutron-separation energy. Journal of Physics G: Nuclear and Particle Physics, 43, [115101]. https://doi.org/10.1088/09543899/43/11/115101

\section{General rights}

Copyright and moral rights for the publications made accessible in the UWS Academic Portal are retained by the authors and/or other copyright owners and it is a condition of accessing publications that users recognise and abide by the legal requirements associated with these rights.

\section{Take down policy}

If you believe that this document breaches copyright please contact pure@uws.ac.uk providing details, and we will remove access to the work immediately and investigate your claim. 


\title{
Dipole Response of the Odd-Proton Nucleus ${ }^{205} \mathrm{Tl}$ up to the Neutron-Separation Energy
}

\author{
N. Benouaret, J. Beller, H. Pai, N. Pietralla, V. Yu. Ponomarev, \\ C. Romig, L. Schnorrenberger, and M. Zweidinger \\ Institut für Kernphysik, Technische Universität Darmstadt, D-64289 Darmstadt, Germany
}

M. Scheck

School of Engineering, University of the West of Scotland, Paisley PA1 2BE, United Kingdom and SUPA, Scottish Universities Physics Alliance, Glasgow G12 8QQ, United Kingdom

J. Isaak and D. Savran

ExtreMe Matter Institute EMMI and Research Division, GSI Helmholtzzentrum für Schwerionenforschung GmbH, D-64291 Darmstadt, Germany and Frankfurt Institute FIAS, D-60438 Frankfurt am Main, Germany

\author{
K. Sonnabend \\ Institut für angewandte Physik, Goethe Universität Frankfurt am Main, D-60438 Frankfurt am Main, Germany \\ R. Raut, ${ }^{*}$ G. Rusev ${ }^{\dagger}$ A. P. Tonchev ${ }^{\ddagger}$ W. Tornow, and H. R. Weller \\ Department of Physics, Duke University, Durham, North Carolina 27708, USA and \\ Triangle Universities Nuclear Laboratory, Durham, North Carolina 27708, USA
}

\section{J. H. Kelley}

Department of Physics, North Carolina State University, Raleigh, North Carolina 27695, USA and Triangle Universities Nuclear Laboratory, Durham, North Carolina 27708, USA

(Dated: September 22, 2016)

\begin{abstract}
The low-lying electromagnetic dipole strength of the odd-proton nuclide ${ }^{205} \mathrm{Tl}$ has been investigated up to the neutron separation energy exploiting the method of nuclear resonance fluorescence. In total, 61 levels of ${ }^{205} \mathrm{Tl}$ have been identified. The measured strength distribution of ${ }^{205} \mathrm{Tl}$ is discussed and compared to those of even-even and even-odd mass nuclei in the same mass region as well as to calculations that have been performed within the quasi-particle phonon model.
\end{abstract}

PACS numbers: 21.10.Re; 21.10.Hw; 23.20.Lv; 25.20.Dc; 21.60.Jz; 27.50.+e

\section{INTRODUCTION}

The low-lying electromagnetic dipole strength of atomic nuclei and the structure of dipole-excited states below the neutron-separation energy have drawn considerable attention in nuclear physics in the past decades. They can be categorized into two groups exhibiting either electric or magnetic radiation character. Examples for nuclear structures

\footnotetext{
*Present address: UGC-DAE Consortium of Scientific Research, Kolkata, India.

${ }^{\dagger}$ Present address: Chemistry Division, Los Alamos National Laboratory, Los Alamos, New Mexico 87545, USA.

${ }^{\ddagger}$ Present address: Nuclear and Chemical Sciences Division, Lawrence Livermore National Laboratory, Livermore, California 94550, USA.
}

carrying significant low-lying electric dipole strength are quadrupole-octupole-coupled two-phonon state, typically occurring at excitation energies below $5 \mathrm{MeV}[1-3]$, and the pygmy dipole resonance (PDR) [4]. The PDR is an accumulation of $J^{\pi}=1^{-}$states which has been observed mainly in magic and semimagic nuclei at excitation energies between 5 and $10 \mathrm{MeV}$. On the other hand, the scissors mode $[5,6]$ and spin-flip excitations [7] represent examples for pronounced magnetic dipole strength. The scissors mode is situated at excitation energies of about 3 $\mathrm{MeV}$ in deformed heavy nuclei, whereas spin-flip excitations typically occur at higher energies, depending on the local shell structure.

A systematic investigation of low-lying electromagnetic dipole strength in nuclei allows to improve our understanding of all of these phenomena. An ideal tool to use in the study of low-lying dipole strength 
is the method of photon scattering or nuclear resonance fluorescence (NRF). In NRF measurements, photons are used to probe nuclear structure. Since real photons allow only for a small angular momentum transfer, mainly dipole excitations are induced in these photon-scattering measurements.

Mostly even-even nuclei, i.e., nuclei with even neutron and proton numbers, have been studied by means of NRF up to the neutron separation threshold, whereas data on even-odd nuclei for excitation energies exceeding $4 \mathrm{MeV}$ only scarcely exist. They include: ${ }^{89} \mathrm{Y}$ (with $N=50$ and $Z=39$ ) [8], ${ }^{139} \mathrm{La}$ (with $N=82$ and $Z=57$ ) [9], ${ }^{207} \mathrm{~Pb}$ (with $N=125$ and $Z=82$ ) [10,11], as well as ${ }^{209} \mathrm{Bi}$ (with $N=126$ and $Z=83$ ) [12]. The main difficulty of NRF measurements on heavy even-odd nuclei is usually the high fragmentation of the strength, resulting in many rather weak excited states that are difficult to observe individually. This unfortunate situation is slightly relaxed in nuclides in the vicinity of shell closures, e.g., for the stable ${ }^{209} \mathrm{Bi}$ nucleus or the Thallium isotopes with one proton outside of the $Z=82$ shell closure.

The PDR was observed for the first time in $(n, \gamma)$ experiments with thermal and fast neutrons (see, e.g. [13]) and resonant photon scattering experiments (see, e.g. [14, 15]). It had a form of a bump on the low-energy tail of the giant dipole resonance. Its energy centroid was found around $5-7 \mathrm{MeV}$ in different nuclei. From systematic studies of neutron capture $\gamma$-ray spectra for $\mathrm{N}=82-126$ region, the energy and strength of the resonance have been found to increase with neutron number $[16,17]$. Poor resolution of $\mathrm{NaI}$ detectors used those days, did not allow to investigate the PDR fine structure. It became possible with Germanium (HPGe) detectors of better resolution and efficiency in modern NRF experiments. In this paper we report the results of this type of experiment on ${ }^{205} \mathrm{Tl}$ enriched target which was never studied before. The $J^{\pi}=1 / 2^{+}$ground state of this $\mathrm{Z}=81$ nucleus is dominated by a hole in the $\pi\left(3 s_{1 / 2}\right)$ subshell below the $\mathrm{Z}=82$ shell closure. With neutron number $N=124$ for ${ }_{81}^{205} \mathrm{Tl}_{124}$, two neutrons are missing from the shell closure at $N=126$.

In the following, the method of NRF with continuous-energy bremsstrahlung is presented followed by experimental results from measurements on a naturally composed $\mathrm{Tl}$ target, as well as on a target highly enriched in ${ }^{205} \mathrm{Tl}$. Subsequently, the results of the present measurements will be compared with other nuclei in this mass region and to calcula- tions that have been performed in the framework of the quasi-particle phonon model (QPM) [18].

\section{METHOD OF NUCLEAR RESONANCE FLUORESCENCE}

The nuclear resonance fluorescence method [19] is based on the resonant absorption of a photon by an atomic nucleus and its subsequent decay back to its ground state or to other lower-lying energy levels. Due to the small momentum transfer of incident photons, mainly dipole and, to a lesser extent, quadrupole transitions are induced. From the observed peak area $A_{i, 0}$ of $\gamma$-ray lines in the spectrum corresponding to a transitions from the state $i$ to the ground state 0 , the energy integrated elastic scattering cross section $I_{i, 0}$ of an excited state at an excitation energy $E_{i}$ is derived:

$$
I_{i, 0}=\frac{A_{i, 0}}{N_{T} N_{\gamma}\left(E_{i}\right) \varepsilon\left(E_{\gamma}\right) W(\theta)} .
$$

Here, $N_{T}$ is the number of target nuclei, $N_{\gamma}$ the absolute photon flux irradiating the target, $\varepsilon$ the absolute efficiency accounting for the intrinsic efficiency as well as the detector geometry, and $W(\theta)$ is the angular distribution of the emitted $\gamma-$ ray. The transition strength quantified by the ground-state width $\Gamma_{0}$ can be extracted from the measured integrated cross section $I_{i, 0}$ :

$$
I_{i, 0}=\pi^{2}\left(\frac{\hbar c}{E_{x}}\right)^{2} g \frac{\Gamma_{0}^{2}}{\Gamma}
$$

where $\Gamma$ is the total decay width and $g=\frac{\left(2 J_{i}+1\right)}{\left(2 J_{0}+1\right)}$ is a spin dependent factor $\left(J_{0}=\frac{1}{2}\right.$ and $J_{i}$ denote the spin quantum numbers of the ground and the excited states of ${ }^{205} \mathrm{Tl}$, respectively). In NRF experiments, the ground-state decay width $\Gamma_{0}$ is fully determined if the branching ratio $\frac{\Gamma_{0}}{\Gamma}$ to the ground state is known, which requires that all branching transitions to intermediate excited states (so-called inelastic transitions) have been observed:

$$
\frac{\Gamma_{0}}{\Gamma}=\frac{1}{1+\sum_{f>0} \frac{\Gamma_{f}}{\Gamma}} .
$$

This determination of branching transitions cannot always be achieved because in NRF experiments using continuous-energy bremsstrahlung, a high radiation background due to non-resonant photon scattering occurs, that increases exponentially towards 
lower energies. Thus, small branching ratios to lower-lying states are difficult to measure and may escape detection. Furthermore, another difficulty is given by the nearly isotropic angular distributions of the photons emitted during the $\gamma$-decay of oddmass nuclei with half-integer spin quantum numbers. As a consequence, an unambiguous assignment of spin quantum numbers to the excited states from the measured angular distributions is only feasible in the case of strong transitions and corresponding high statistics.

Without knowledge of the spin quantum number $J_{i}$ of the excited state, the product $g \frac{\Gamma_{0}^{2}}{\Gamma}$ can be unambiguously derived from the measured integrated scattering cross section. This allows for the determination of the reduced $B(E 1) \uparrow$ and $B(M 1) \uparrow$ excitation probabilities:

$$
\begin{gathered}
B(E 1) \uparrow=0.9554\left(\frac{g \Gamma_{0}}{E_{\gamma}^{3}}\right)\left(10^{-3} \mathrm{e}^{2} \mathrm{fm}^{2}\right), \\
B(M 1) \uparrow=0.0864\left(\frac{g \Gamma_{0}}{E_{\gamma}^{3}}\right)\left(\mu_{N}^{2}\right),
\end{gathered}
$$

with the ground-state transition width $\Gamma_{0}$ in $\mathrm{meV}$ and the transition energy $E_{\gamma}$ in $\mathrm{MeV}$, if information on the ground-state decay branching ratio $\frac{\Gamma_{0}}{\Gamma}$ is available.

In the case of an unknown branching ratio $\frac{\Gamma_{0}}{\Gamma}$ to the ground state, which is by definition smaller or equal to unity, only a lower limit of $g \Gamma_{0}$ (assuming no transitions to intermediate states, i.e., $\Gamma=\Gamma_{0}$ ) and, consequently, of $B(E 1) \uparrow$ or $B(M 1) \uparrow$ can be deduced.

\section{EXPERIMENTS}

Two nuclear resonance fluorescence experiments, one on metallic, naturally composed Thallium (2060.0 mg) and one on a target enriched to $99.9 \%$ in ${ }^{205} \mathrm{Tl}(1938.4 \mathrm{mg})$ have been performed at the Darmstadt High Intensity Photon Setup (DHIPS) [20] at the Darmstadt superconducting electron linear accelerator S-DALINAC at Technische Universität Darmstadt. The natural abundance of ${ }^{203} \mathrm{Tl}$ accounts to $29.5 \%$, the one of ${ }^{205} \mathrm{Tl}$ to $70.5 \%$. The comparison of both measurements allows for an identification of transitions of ${ }^{203} \mathrm{Tl}$ next to those of ${ }^{205} \mathrm{Tl}$.

Both targets have been irradiated for about 80 hours by an unpolarized bremsstrahlung beam with an endpoint energy of $7.5 \mathrm{MeV}$. The neutronseparation energies of ${ }^{205} \mathrm{Tl}$ and ${ }^{203} \mathrm{Tl}$ are 7.546 and $7.850 \mathrm{MeV}$, respectively. The corresponding protonseparation energies are 6.419 and $5.704 \mathrm{MeV}$, respectively. The bremsstrahlung has been generated by stopping a mono-energetic electron beam of $7.5 \mathrm{MeV}$ kinetic energy and an average current of $16 \mu \mathrm{A}$ and $31 \mu \mathrm{A}$, respectively, in a copper radiator. It reaches the NRF target after having passed through a collimator system made out of copper. The endpoint energy was chosen lower than the respective neutron separation energies of ${ }^{203} \mathrm{Tl}$ and ${ }^{205} \mathrm{Tl}$ in order to avoid background radiation resulting from $(\gamma, n)$ or $\left(n, n^{\prime} \gamma\right)$ inelastic neutron scattering reactions, which in turn induce $(n, \gamma)$ neutron capture reactions in the measuring setup. Both targets were sandwiched between two boron disks with a total mass of 240.8 $\mathrm{mg}$ (naturally composed) and $394.3 \mathrm{mg}$ (enriched to $99.5 \%$ in ${ }^{11} \mathrm{~B}$ ), respectively. The well-known transitions of ${ }^{11} \mathrm{~B}$ (NNDC, 2007) are used to calibrate the energy of the recorded spectra as well as the absolute photon flux during the measurements.

The scattered photons were counted by three High Purity Germanium (HPGe) detectors with efficiencies of $100 \%$ relative to a standard $\mathrm{NaI}$ detector, and mounted at polar angles of $90^{\circ}, 95^{\circ}$, and $130^{\circ}$ relative to the incident beam, and located at a distance of about $25 \mathrm{~cm}$ to the NRF target. The entire detector-NRF-target system was mounted in a lead cave to shield from the background radiation produced at the radiator. In order to improve the signal-to-background ratio, the detectors were surrounded by Bismuth Germanate (BGO) scintillation detectors, which work as Compton- and escapesuppression shields. Copper and lead absorbers were placed in front of each detector for reducing the lowenergy part of the non-resonant background radiation.

The absolute efficiencies of the detectors have been determined using a ${ }^{56}$ Co source as well as simulations with the GEANT4 Monte Carlo simulation tool kit [21] taking the detector geometry into account.

The well-known transitions of ${ }^{11} \mathrm{~B}$ (NNDC, 2007) are used to calibrate the energy of the recorded spectra as well as the product of photon flux and efficiency $N_{\gamma} \varepsilon\left(E_{\gamma}\right)$ during the measurements.

\section{RESULTS AND DISCUSSION}

Spectra of the scattered photons off the naturally composed (upper panel) and the enriched thallium (lower panel) targets, respectively, recorded at 


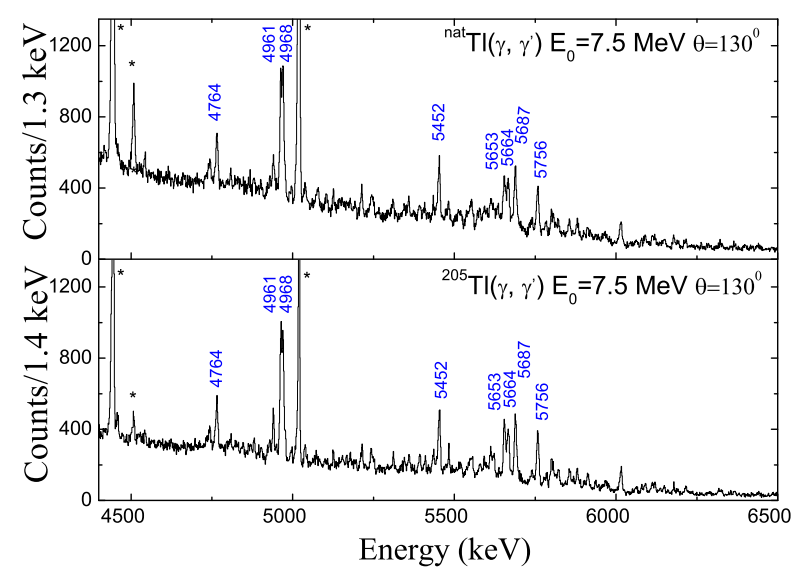

FIG. 1: Part of the photo-excitation spectrum of ${ }^{\text {nat }} \mathrm{Tl}$ (upper panel) and ${ }^{205} \mathrm{Tl}$ (lower panel) measured at $130^{\circ}$ at an end-point energy of $7.5 \mathrm{MeV}$ with a natural and enriched thallium target, respectively. Peaks marked by asterisks are attributed to transitions of ${ }^{11} \mathrm{~B}$ and corresponding escape lines.

DHIPS are shown in Fig. 1. Besides transitions originating from the ${ }^{11} \mathrm{~B}\left(\gamma, \gamma^{\prime}\right)$ reaction, a concentration of transitions from ${ }^{205} \mathrm{Tl}$ is visible in the energy range between $4.5 \mathrm{MeV}$ and $6.5 \mathrm{MeV}$. The spectra of both targets are very similar, already indicating that most of the strong transitions can be assigned to ${ }^{205} \mathrm{Tl}$. In this manuscript we restrict ourselves to the analysis of the resolved $\gamma$-ray lines which we consider as justified for this nucleus in close vicinity to the double shell closure of ${ }^{208} \mathrm{~Pb}$.

In total, 61 transitions have been assigned to ${ }^{205} \mathrm{Tl}$ from our comparison of the $\gamma$-ray intensities originating from the isotopically enriched and from the naturally composed $\mathrm{Tl}$ targets. Two transitions can be attributed to ${ }^{203} \mathrm{Tl}$ based on their abscence in the $\gamma$-ray spectra taken with the sample enriched in the isotope ${ }^{205} \mathrm{Tl}$. Table I provides an overview of the observed transitions of ${ }^{205} \mathrm{Tl}$ using the $\left(\gamma, \gamma^{\prime}\right)$ reaction at an endpoint energy of $7.5 \mathrm{MeV}$. Here, it is assumed that all observed transitions correspond to the direct decay of excited states with excitation energy $E_{x}$ back to the ground state. However, this must not always be the case, as will be discussed in detail below in Sec. IV B, since an observed transition may also correspond to the decay to an intermediate state, such as the first excited $\frac{3}{2}^{+}$level at $203.7 \mathrm{keV}$ excitation energy. As indicated above, due to the low momentum transfer of real photons, the observed transitions should mostly have a dipole character (either $E 1$ or $M 1$ ) corresponding to the excitation from the $\frac{1}{2}^{+}$ground state to levels with spin quantum numbers $\frac{1}{2}$ or $\frac{3}{2}$ with either positive or negative parity quantum numbers. Therefore, the reduced $B(E 1) \uparrow$ and $B(M 1) \uparrow$ transition probabilities are given, assuming dipole character for all observed transitions besides the photon-scattering cross section as the primary observable. The listed values represent an error-weighted average of the results from measurements with natural and enriched targets. The same information concerning the two transitions identified for ${ }^{203} \mathrm{Tl}$ is given in Table II.

In the tables, only transitions exceeding the detection limit of the present experiments are considered. The experimental energy-dependent sensitivity limit has been chosen according to Ref. [22]. It is based on the background present in the spectra and requires the relative uncertainty of the observed peak areas to be smaller than $30 \%$ to be taken into account.

In the following, the observed transitions will be discussed.

\section{A. Spin Quantum Numbers}

As has been indicated in Eq. (2), a spin quantum number assignment to the photo-excited levels $J=\left(\frac{1}{2}, \frac{3}{2}\right.$ or $\left.\frac{5}{2}\right) \hbar$ is crucial for the determination of the ground-state transition width $\Gamma_{0}$. The spin quantum number can be deduced from the angular distribution ratio $\frac{W\left(90^{\circ}\right)}{W\left(130^{\circ}\right)}$ which amounts to $0.85,1$, or 1.15 for a spin sequence of $\frac{1}{2} \rightarrow \frac{3}{2} \rightarrow \frac{1}{2}$, $\frac{1}{2} \rightarrow \frac{1}{2} \rightarrow \frac{1}{2}$, and $\frac{1}{2} \rightarrow \frac{5}{2} \rightarrow \frac{1}{2}$, respectively. The experimental angular distribution ratios are obtained from the intensity ratios of the transitions measured at simultaneously at scattering angles $90^{\circ}$ and $130^{\circ}$, respectively.

As can be seen in Fig. 2, within the experimental uncertainties of the angular distribution ratio, it is difficult to unambiguously assign a spin quantum number to the photo-excited states due to the large statistical uncertainties in most cases. However, dipole rather than quadrupole character may be assigned to the excited states at excitation energies higher than $E_{x}=5.25 \mathrm{MeV}$. There, the angular distribution ratios tend to be smaller than unity indicating either $J=\frac{1}{2}$ or $J=\frac{3}{2}$ for the excited states. 
TABLE I: Properties of the photo-excited levels identified in ${ }^{205} \mathrm{Tl}$ using the $\left(\gamma, \gamma^{\prime}\right)$ reaction at an end-point energy of $7.5 \mathrm{MeV}$ with the corresponding excitation energies $E_{x}$, angular distribution ratios, the measured integrated elastic scattering cross sections $I_{i, 0}$, the extracted product of the statistical factor $\mathrm{g}$ and the transition width ratios $\Gamma_{0}{ }^{2} / \Gamma$ and the reduced excitation probabilities $B(E 1) \uparrow$ or $B(M 1) \uparrow$ for excited states with either negative or positive parity quantum number, respectively.

\begin{tabular}{|c|c|c|c|c|c|}
\hline $\begin{array}{c}E_{x} \\
(\mathrm{keV})\end{array}$ & $\frac{W\left(90^{\circ}\right)}{W\left(130^{\circ}\right)}$ & $\begin{array}{c}I_{i, 0} \\
(\mathrm{eV} \mathrm{b})\end{array}$ & $\begin{array}{l}g \cdot \frac{\Gamma_{0}^{2}}{\Gamma} \\
(\mathrm{eV})\end{array}$ & $\begin{array}{c}B(E 1) \uparrow \\
\left(10^{-3} \mathrm{e}^{2} \mathrm{fm}^{2}\right)\end{array}$ & $\begin{array}{c}B(M 1) \uparrow \\
\left(\mu_{N}^{2}\right)\end{array}$ \\
\hline $4000.6(2)$ & $1.26(21)$ & $78(14)$ & $0.32(6)$ & $4.83(90)$ & $0.44(8)$ \\
\hline $4159.9(2)$ & $0.79(11)$ & $99(22)$ & $0.44(10)$ & $5.9(13)$ & $0.53(12)$ \\
\hline $4262.5(4)$ & $1.33(26)$ & $58(12)$ & $0.28(6)$ & $3.40(68)$ & $0.31(6)$ \\
\hline $4341.9(5)^{b}$ & $1.02(36)$ & $24(5)$ & $0.12(2)$ & $1.38(29)$ & $0.12(3)$ \\
\hline $4348.4(4)^{b, d, e}$ & & $30(5)$ & $0.15(2)$ & $1.70(28)$ & $0.15(3)$ \\
\hline $4731.6(7)^{a, b}$ & $1.29(57)$ & $17(4)$ & $0.10(2)$ & $0.89(21)$ & $0.08(2)$ \\
\hline $4741.4(9)$ & $0.76(17)$ & $61(12)$ & $0.36(7)$ & $3.21(64)$ & $0.29(6)$ \\
\hline $4828.1(11)$ & $1.25(48)$ & $27(7)$ & $0.16(4)$ & $1.37(36)$ & $0.12(3)$ \\
\hline $4878.4(4)^{a}$ & $1.20(38)$ & $34(6)$ & $0.21(4)$ & $1.76(32)$ & $0.16(3)$ \\
\hline $4926.5(6)$ & $1.23(30)$ & $48(9)$ & $0.30(6)$ & $2.44(45)$ & $0.22(4)$ \\
\hline $4938.2(2)^{a}$ & $1.19(17)$ & $86(11)$ & $0.55(7)$ & $4.35(57)$ & $0.39(5)$ \\
\hline $4947.0(10)$ & $1.21(37)$ & $40(8)$ & $0.25(5)$ & $2.00(42)$ & $0.18(4)$ \\
\hline $4961.1(2)^{c}$ & $0.87(16)$ & $312(55)$ & $2.00(35)$ & $15.7(27)$ & $1.73(32)$ \\
\hline $4967.8(1)^{a, c}$ & $0.93(10)$ & $382(71)$ & $2.46(45)$ & $19.1(35)$ & $1.72(23)$ \\
\hline 4975.1(6) & $1.03(17)$ & $72(7)$ & $0.46(5)$ & $3.59(35)$ & $0.32(3)$ \\
\hline $4994.1(3)$ & $0.95(36)$ & $41(10)$ & $0.27(7)$ & $2.05(52)$ & $0.19(5)$ \\
\hline $5007.5(6)$ & $1.14(32)$ & $42(7)$ & $0.28(5)$ & $2.10(35)$ & $0.19(3)$ \\
\hline $5036.5(6)$ & $0.89(23)$ & $58(12)$ & $0.39(8)$ & $2.88(60)$ & $0.26(5)$ \\
\hline $5071.4(5)^{b, d}$ & $1.11(37)$ & $31(5)$ & $0.21(4)$ & $1.50(26)$ & $0.14(2)$ \\
\hline $5123.8(5)$ & $0.76(32)$ & $48(11)$ & $0.33(7)$ & $2.33(51)$ & $0.21(5)$ \\
\hline $5164.6(7)^{d}$ & $1.27(31)$ & $39(7)$ & $0.27(5)$ & $1.88(34)$ & $0.17(3)$ \\
\hline $5211.8(6)$ & $0.77(33)$ & $81(19)$ & $0.57(13)$ & $3.86(89)$ & $0.35(8)$ \\
\hline $5240.4(7)$ & $0.66(20)$ & $52(15)$ & $0.37(11)$ & $2.47(70)$ & $0.22(6)$ \\
\hline $5308.6(4)^{a}$ & $0.94(25)$ & $50(17)$ & $0.37(13)$ & $2.36(80)$ & $0.21(7)$ \\
\hline $5343.6(9)^{a, b}$ & $0.99(43)$ & $39(9)$ & $0.29(6)$ & $1.82(40)$ & $0.17(4)$ \\
\hline $5357.3(5)$ & $0.84(13)$ & $76(18)$ & $0.57(14)$ & $3.52(85)$ & $0.32(8)$ \\
\hline $5390.9(4)$ & $0.90(21)$ & $72(14)$ & $0.54(11)$ & $3.31(65)$ & $0.30(6)$ \\
\hline $5406.6(8)^{b}$ & $0.82(20)$ & $48(7)$ & $0.33(5)$ & $2.00(33)$ & $0.18(3)$ \\
\hline $5432.9(6)$ & $0.74(18)$ & $67(12)$ & $0.51(9)$ & $3.06(54)$ & $0.28(5)$ \\
\hline $5451.2(5)^{a}$ & $0.98(9)$ & $280(35)$ & $2.16(27)$ & $12.8(16)$ & $1.15(14)$ \\
\hline $5480.2(5)^{b}$ & $0.89(17)$ & $90(17)$ & $0.70(14)$ & $4.07(79)$ & $0.37(7)$ \\
\hline $5552.6(6)^{a, b}$ & $1.34(32)$ & $86(30)$ & $0.69(24)$ & $3.8(14)$ & $0.35(12)$ \\
\hline $5577.1(7)^{a, b}$ & $1.42(49)$ & $43(11)$ & $0.35(9)$ & $1.90(48)$ & $0.17(4)$ \\
\hline 5589.6(9) & $0.76(13)$ & $84(18)$ & $0.68(15)$ & $3.74(79)$ & $0.34(7)$ \\
\hline $5598.1(8)^{a, b}$ & $0.96(47)$ & $47(14)$ & $0.39(12)$ & $2.10(63)$ & $0.19(6)$ \\
\hline $5610.4(5)$ & $0.87(21)$ & $124(25)$ & $1.02(21)$ & $5.5(11)$ & $0.50(10)$ \\
\hline $5619.8(7)$ & $0.94(18)$ & $107(11)$ & $0.88(9)$ & $4.74(49)$ & $0.43(4)$ \\
\hline $5652.3(5)$ & $0.82(8)$ & $274(44)$ & $2.28(36)$ & $12.1(19)$ & $1.09(17)$ \\
\hline $5664.7(6)^{a}$ & $0.94(14)$ & $207(19)$ & $1.73(16)$ & $9.08(85)$ & $0.82(8)$ \\
\hline $5686.2(3)$ & $0.89(8)$ & $337(55)$ & $2.84(46)$ & $14.8(24)$ & $1.34(22)$ \\
\hline $5693.3(9)$ & $0.92(34)$ & $67(15)$ & $0.57(13)$ & $2.95(66)$ & $0.27(6)$ \\
\hline $5737.6(8)$ & $0.90(17)$ & $79(9)$ & $0.68(8)$ & $3.44(39)$ & $0.31(4)$ \\
\hline $5755.8(3)$ & $0.89(7)$ & $325(30)$ & $2.81(26)$ & $14.1(13)$ & $1.27(12)$ \\
\hline $5781.4(6)$ & $0.86(20)$ & $63(19)$ & $0.55(16)$ & $2.73(81)$ & $0.25(7)$ \\
\hline $5797.8(9)$ & $0.76(15)$ & $107(17)$ & $0.94(15)$ & $4.59(73)$ & $0.41(7)$ \\
\hline $5803.8(9)^{a}$ & $0.79(28)$ & $63(14)$ & $0.55(12)$ & $2.70(59)$ & $0.24(5)$ \\
\hline $5811.6(9)$ & $1.12(40)$ & $44(9)$ & $0.39(8)$ & $1.90(37)$ & $0.17(3)$ \\
\hline $5819.7(4)$ & $1.07(15)$ & $105(11)$ & $0.93(9)$ & $4.50(46)$ & $0.41(4)$ \\
\hline $5864.7(9)$ & $0.92(26)$ & $45(7)$ & $0.40(6)$ & $1.91(30)$ & $0.17(3)$ \\
\hline $5878.1(5)$ & $0.81(10)$ & $116(22)$ & $1.05(20)$ & $4.93(93)$ & $0.45(8)$ \\
\hline $5910.5(6)$ & $0.93(13)$ & $79(11)$ & $0.72(10)$ & $3.32(48)$ & $0.30(4)$ \\
\hline $5963.8(18)$ & $0.69(14)$ & $63(14)$ & $0.58(13)$ & $2.61(60)$ & $0.24(5)$ \\
\hline $6060.7(4)$ & $0.86(17)$ & $48(7)$ & $0.46(7)$ & $1.96(29)$ & $0.18(3)$ \\
\hline $6088.5(5)$ & $0.74(15)$ & $80(16)$ & $0.77(16)$ & $3.26(66)$ & $0.29(6)$ \\
\hline $6109.4(8)$ & $0.70(23)$ & $65(16)$ & $0.63(15)$ & $2.63(65)$ & $0.24(6)$ \\
\hline $6146.8(9)$ & $0.82(13)$ & $68(10)$ & $0.67(10)$ & $2.77(39)$ & $0.25(4)$ \\
\hline $6176.6(4)^{d}$ & $0.87(19)$ & $58(8)$ & $0.58(8)$ & $2.34(32)$ & $0.21(3)$ \\
\hline $6188.9(6)^{d}$ & $0.82(21)$ & $47(8)$ & $0.47(8)$ & $1.90(33)$ & $0.17(3)$ \\
\hline $6213.3(9)^{d}$ & $0.51(15)$ & $46(17)$ & $0.47(17)$ & $1.85(70)$ & $0.17(6)$ \\
\hline $6315.2(10)^{d}$ & $0.85(26)$ & $37(7)$ & $0.39(7)$ & $1.46(26)$ & $0.13(2)$ \\
\hline $6364.6(6)^{d}$ & $1.01(29)$ & $41(6)$ & $0.43(7)$ & $1.61(25)$ & $0.15(2)$ \\
\hline
\end{tabular}

${ }^{a}$ Single escape contribution subtracted

${ }^{b}$ Possible branching transition; see Table IV

${ }^{c} \mathrm{E} 1$ and $\mathrm{M} 1$ not corrected by the branching transition

${ }^{d}$ observed in enriched target only

${ }^{e}$ observed in enriched target in one detector only 
TABLE II: Properties of the photo-excited levels identified in ${ }^{203} \mathrm{Tl}$ using the $\left(\gamma, \gamma^{\prime}\right)$ reaction and a bremsstrahlung end-point energy of $7.5 \mathrm{MeV}$ with the corresponding excitation energies $E_{x}$, angular distribution ratios, the measured integrated elastic scattering cross sections $I_{i, 0}$, the extracted product of the statistical factor $\mathrm{g}$ and the transition width ratios $\Gamma_{0}^{2} / \Gamma$ and the reduced excitation probabilities.

\begin{tabular}{cccccc}
\hline \hline $\begin{array}{c}E_{x} \\
(\mathrm{keV})\end{array}$ & $\frac{W\left(90^{\circ}\right)}{W\left(130^{\circ}\right)}$ & $\begin{array}{c}I_{i, 0} \\
(\mathrm{eV} \mathrm{b})\end{array}$ & $\begin{array}{c}g \cdot \frac{\Gamma_{0}^{2}}{\Gamma} \\
(\mathrm{eV})\end{array}$ & $\begin{array}{c}B(E 1) \uparrow \\
\left(10^{-3} \mathrm{e}^{2} \mathrm{fm}^{2}\right)\end{array}$ & $\begin{array}{c}B(M 1) \uparrow \\
\left(\mu_{N}^{2}\right)\end{array}$ \\
\hline $5076.5(4)$ & $0.84(21)$ & $152(22)$ & $1.02(15)$ & $7.5(11)$ & $0.68(10)$ \\
$5102.3(4)$ & $0.89(21)$ & $126(16)$ & $0.86(11)$ & $6.16(80)$ & $0.56(7)$ \\
\hline \hline
\end{tabular}

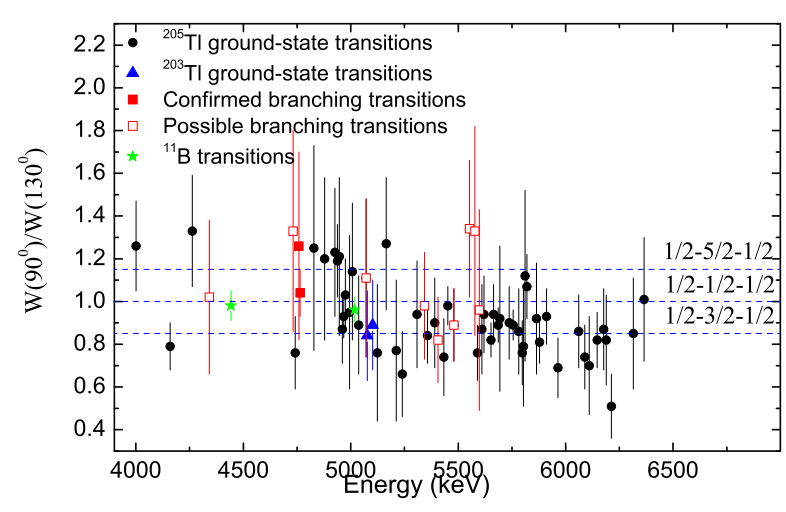

FIG. 2: Measured angular distribution ratios $\frac{W\left(90^{\circ}\right)}{W\left(130^{\circ}\right)}$ of the observed $\gamma$-ray transitions in ${ }^{203,205} \mathrm{Tl}$. Confirmed and candidates for inelastic transitions are indicated by full and open squares, respectively. ${ }^{11} \mathrm{~B}$ ground-state transitions are marked by stars.

\section{B. Decay Pattern}

Usually, a photo-excited state can decay via various decay channels. First of all, it can directly decay back to the ground state. In accordance with classical scattering reactions, this situation is often referred to as elastic photon scattering. On the other hand, the excited state can decay via intermediate states. This case is correspondingly called inelastic photon scattering.

As has already been pointed out above, peaks observed in the measured $\mathrm{Tl}$ spectra do not always correspond to direct decays to the ground state, i.e., to elastic transitions, but may stem instead from inelastic transitions to intermediate states. The Ritz variation principle allows us to check whether a transition may be of inelastic character or not. Applying the Ritz principle, the energy difference of two $\gamma$-ray transitions $E_{\gamma, 1}$ and $E_{\gamma, 2}$ is compared with the energies of low-lying states, e.g., the one of the $\frac{3}{2}_{1}^{+}$ level. If they match, the excited level at an excitation energy $E_{\gamma, 1}$ may decay also via the low-lying state resulting in an inelastic transition peak at $E_{\gamma, 2}$. Nevertheless, the energies may also coincide by accident making analysis of this criterion necessary but not sufficient for the assignment of inelastic transitions.

According to the Ritz principle, 09 out of 61 observed transitions of ${ }^{205} \mathrm{Tl}$ may be considered as inelastic transitions of the photo-excited levels to the well-known low-lying levels located at excitation energies $E_{x}$ of $203.7 \mathrm{keV}\left(J^{\pi}=3 / 2^{+}\right)$, $619.22 \mathrm{keV}\left(J^{\pi}=5 / 2^{+}\right), 1140.99 \mathrm{keV}\left(J^{\pi}=3 / 2^{+}\right)$, $1179.98 \mathrm{keV}\left(J^{\pi}=3 / 2^{+}, 5 / 2^{+}\right), 1219.15 \mathrm{keV}\left(J^{\pi}=\right.$ $\left.1 / 2^{+}\right), 1438.42 \mathrm{keV}\left(J^{\pi}=1 / 2,3 / 2,5 / 2^{+}\right)$, and $1574.03 \mathrm{keV}\left(J^{\pi}=3 / 2^{+}, 5 / 2^{+}\right)$.

The most pronounced transitions of ${ }^{205} \mathrm{Tl}$ have been observed at $\gamma$-ray energies of $4961.1 \mathrm{keV}$ and $4967.8 \mathrm{keV}$, respectively. Following the Ritz principle, the peaks observed in the spectra at $4764.1 \mathrm{keV}$ and $4759.3 \mathrm{keV}$, respectively could be associated with transitions from the $4961.1 \mathrm{keV}$ and $4967.8 \mathrm{keV}$ levels to the $E_{x}=203.7 \mathrm{keV}$ first excited state, respectively.

Therefore, in order to decide whether the deexcitations correspond to branching transitions or to ground-state transitions, the present experiments have been complemented by an additional measurement carried out at the High Intensity $\gamma$-ray Source $(\mathrm{HI} \vec{\gamma} \mathrm{S})[23]$ at the Triangle Universities Nuclear Laboratory in Durham, NC, USA. There, the natural Thallium target has been exposed to a nearly monoenergetic linearly polarized $\gamma$-ray beam (FWHM $3 \%)$. At $\mathrm{HI} \vec{\gamma} \mathrm{S}$, the photon beam is produced via Compton backscattering of laser photons generated in a free electron laser. For a detailed description of $\mathrm{HI} \vec{\gamma} \mathrm{S}$ we refer to Ref. [23].

The $\gamma$-rays scattered from the target have been counted by four HPGe detectors with efficiencies of $60 \%$ for three detectors and $20 \%$ for one detector 


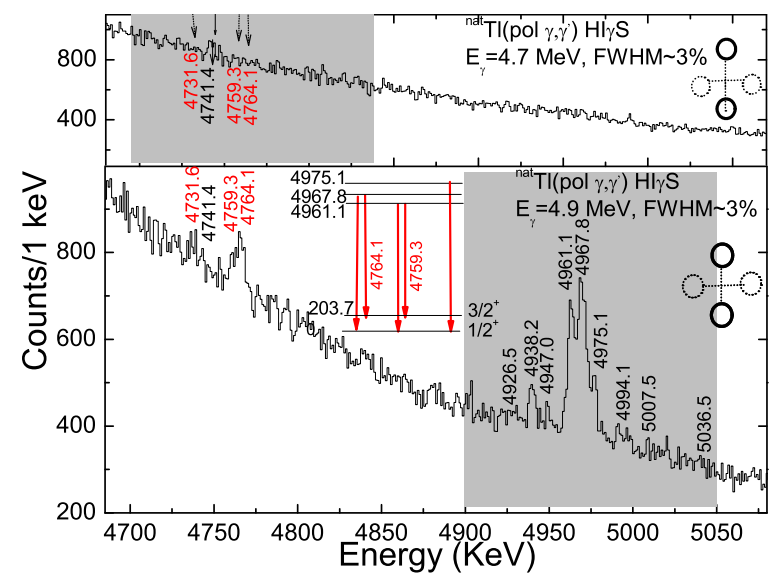

FIG. 3: Photon-scattering spectrum of ${ }^{\text {nat }} \mathrm{Tl}$ at mean polar angle $\theta=90^{\circ}$ using a mono-energetic polarized incident photon beam at $4.7 \mathrm{MeV}$ (upper part) and $4.9 \mathrm{MeV}$ (lower part) at $\mathrm{HI} \vec{\gamma} \mathrm{S}$. The gray shaded area marks the energy spread of the incident photon beam (FWHM 3\%)

relative to a standard NaI detector. They have been mounted around the target at polar angles perpendicular to the incoming beam. The photon beam has been tuned to $4.7 \mathrm{MeV}$ and $4.9 \mathrm{MeV}$ mean beam energy $E_{b}$. The measurements at both energies lasted for about three hours each. Figure 3 shows the sum of the recorded spectra of two vertical detectors for the measurements at $E_{b}=4.7 \mathrm{MeV}$ (upper panel) and at $E_{b}=4.9 \mathrm{MeV}$ (lower panel), respectively. In the measurement at $E_{b}=4.7 \mathrm{MeV}$ no peaks were observed at either $4764 \mathrm{keV}$ or 4759 $\mathrm{keV}$, only a weak previuosly known state was seen at $4741.4 \mathrm{keV}$. However, in the second measurement at $E_{b}=4.9 \mathrm{MeV}$, along with the states at 4961.1 $\mathrm{keV}$ and $4967.8 \mathrm{keV}$ excitation energy populated by the incident $\gamma$-ray beam, transitions at $4764 \mathrm{keV}$ and $4759 \mathrm{keV}$ were observed. The corresponding peaks are thus associated with decay branches of the levels at $4961 \mathrm{keV}$ and $4967 \mathrm{keV}$, respectively to the first excited state with $J^{\pi}=3 / 2^{+}$. Thus, there is no excited state of ${ }^{205} \mathrm{Tl}$ at $4764 \mathrm{keV}$ or $4759 \mathrm{keV}$. However the possible decay branch of the levels at 4961.1 $\mathrm{keV}$ and $4967.8 \mathrm{keV}$ into the second excited state of ${ }^{205} \mathrm{Tl}$ at $601.4 \mathrm{keV}$ excitation energy, which coincides with the weak transitions at $4341.9 \mathrm{keV}$ and $4348.4 \mathrm{keV}$, respectively, observed at S-DALINAC, have not been observed in $\mathrm{HI} \vec{\gamma} \mathrm{S}$ experiments at a beam energy of $E_{b}=4.9 \mathrm{MeV}$.

Among the levels populated in the measurements at $E_{b}=4.9 \mathrm{MeV}$, one can well distinguish in the recorded spectrum three other populated levels at 4938.2, 4947.0 and $4975.1 \mathrm{keV}$ excitation energy which have also been identified in S-DALINAC measurements. In this spectrum, a transition at 4731 $\mathrm{keV}$, which corresponds to a decay branch of the level at $4938.2 \mathrm{keV}$ to the first excited state at $E_{x}=203.7 \mathrm{keV}$, has been found but $10 \%$ lower than the sensitivity limit. This transition, previously detected at S-DALINAC, has not been found in the measurements at $E_{b}=4.7 \mathrm{MeV}$, which thus could confirm its inelastic character. For the 4947.0 and $4975.1 \mathrm{keV}$ levels, no branching decay has been observed. The transition found at $4741.4 \mathrm{keV}$, which coincides with the deexcitation energy of the level at $4947.0 \mathrm{keV}$ to the first excited state, has been confirmed as a ground-state transition in the measurements at $E_{b}=4.7 \mathrm{MeV}$. The spectrum obtained at $\mathrm{HI} \vec{\gamma} \mathrm{S}$ at $E_{b}=4.9 \mathrm{MeV}$ shows additional weakly populated levels in the energy region of the incident photon beam, indicated with a grey color panel, at 4926.5, 4994.1 and $5007.5 \mathrm{keV}$ excitation energy, respectively. The corresponding peaks observed also at S-DALINAC are thus considered as ground-state transitions. Whereas two branching transitions were assigned to the two photo-excited levels at excitation energies of 4961.1 and $4967.8 \mathrm{keV}$ (see Table III), inelastic character of 12 remaining transitions cannot be confirmed nor excluded. Details of these 12 photo-excited levels which exhibit possible branching transitions to lower-lying excited states are listed in Table IV. The candidate branching transitions exclude transitions for which the corresponding strength is higher than the one of the ground-state transition strength. Indeed, this is noticeable for the $4961.1 \mathrm{keV}$ and $4967.8 \mathrm{keV}$ levels, where the ground-state transitions amount to $70 \%$ of the total decay width. Using monochromatic gamma beams produced from thermal-neutron capture reactions, A. Wolf et al.[24] measured g.s branching ratios of $56 \%$ and $58 \%$ of the photo-excited levels at $7252 \mathrm{keV}$ and $7646 \mathrm{keV}$ of ${ }^{205} \mathrm{Tl}$, respectively accounting for all observed branching. This is consistent with the values measured in our experiments in this excitation energy region and also with those which can be deduced from data on the neighboring nucleus ${ }^{207} \mathrm{~Pb}$ [10]. Furthermore, measurements in the case of ${ }^{60} \mathrm{Ni}$ [25], ${ }^{94} \mathrm{Mo}$ [26], ${ }^{140} \mathrm{Ce}$ [27] have demonstrated that the ground-state decays exceed $50 \%$ of the total decay in the vicinity of the neutron threshold energy. 
TABLE III: Properties of photo-excited states with firmly assigned branching transitions. Given are the level excitation energies $E_{x}$, the transition energies $E_{\gamma}$ of both the elastic and inelastic transitions, the ground-state branching ratios $\frac{\Gamma_{0}}{\Gamma}$ considering only the branching observed in S-DALINAC experiments and confirmed in HI $\vec{\gamma} \mathrm{S}$ measurements, the ground-state widths $g \cdot \Gamma_{0}$ and the $B(E 1) \uparrow, B(M 1) \uparrow$ transition probabilities.

\begin{tabular}{ccccccc}
\hline \hline $\begin{array}{c}E_{x} \\
(\mathrm{keV})\end{array}$ & $\begin{array}{c}E_{\gamma} \\
(\mathrm{keV})\end{array}$ & $\begin{array}{c}E_{f} \\
(\mathrm{keV})\end{array}$ & $\frac{\Gamma_{0}}{\Gamma}$ & $\begin{array}{c}g \Gamma_{0} \\
(\mathrm{eV})\end{array}$ & $\begin{array}{c}B(E 1) \\
\left(10^{-3} \mathrm{e}^{2} \mathrm{fm}^{2}\right)\end{array}$ & $\begin{array}{c}B(M 1) \\
\left(\mu^{2}\right)\end{array}$ \\
\hline \multirow{2}{*}{$4961.1(2)$} & $4961.1(2)$ & 0 & $0.85(3)$ & $2.79(50)$ & $18.5(33)$ & $1.67(30)$ \\
& $4759.3(7)$ & 203.7 & & & & \\
$4967.8(1)$ & $4967.8(1)$ & 0 & $0.71(2)$ & $4.93(92)$ & $27.1(51)$ & $2.45(46)$ \\
& $4764.1(4)$ & 203.7 & & & & \\
\hline
\end{tabular}

\section{Comparison to Neighboring Nuclei}

Figure 4 shows the measured dipole-strength distribution in terms of

$$
g \Gamma_{0}^{\mathrm{red}}=g \frac{\Gamma_{0}}{E_{x}^{3}}
$$

[which is proportional to the reduced transition probabilities, see Eqs. (4) and (5)] for ${ }^{205} \mathrm{Tl}$ from the present measurements assuming $\Gamma=\Gamma_{0}$ in cases where no inelastic transitions have been assigned from the Ritz combination principle in comparison to data on neighboring nuclei near the $N=126$ shell closure.

The results for ${ }^{205} \mathrm{Tl}$ are presented as discrete lines in the second panel of Figure 4. Corresponding results from previous NRF measurements in neighboring even-even ${ }^{204-208} \mathrm{~Pb}$ and odd mass nuclei are shown in other panels for comparison. In all of these isotopes one finds two regions of strength concentration: the first one is located between 4.7 and 5.0 $\mathrm{MeV}$ and the second, broader one, around 5.5 to 6.0 $\mathrm{MeV}$. The observed strength in double-magic ${ }^{208} \mathrm{~Pb}$ and its neighbor ${ }^{207} \mathrm{~Pb}$ is concentrated only in a few excited states in ${ }^{208} \mathrm{~Pb}$ with comparatively large individual strength. In contrast to this, in other $\mathrm{Pb}$ isotopes as well as in ${ }^{205} \mathrm{Tl}$ and ${ }^{209} \mathrm{Bi}$, the strength is significantly more fragmented, i.e. it is distributed over many more weakly excited states. One can notice that the detectable transition strength above the NRF sensitivity limit increases with the difference $(\mathrm{N}-\mathrm{Z})$ which is the strongest in the case of the closed shell ${ }^{208} \mathrm{~Pb}$. This feature of PDR mode has been also observed in other neutron shell closed nuclei. The comparison of the dipole strengths distribution in ${ }^{205} \mathrm{Tl}$ obtained from NRF experiments is done with those of the neighboring nuclei where the

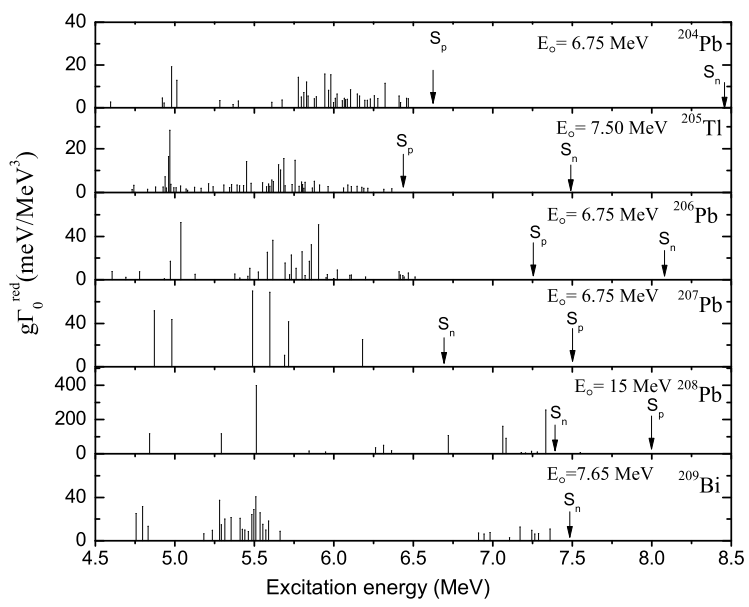

FIG. 4: Systematics of the dipole-strength distributions in stable nuclei near the $N=126$ shell closure observed in NRF experiments using initial electron beams of kinetic energy $E_{0}$ for the bremsstrahlung production. For all nuclei the reduced transition width $g \cdot \Gamma_{0}^{\text {red }}$ is plotted as function of the excitation energy. Data for ${ }^{204,206,207} \mathrm{~Pb}$ are taken from Ref. [10], for ${ }^{208} \mathrm{~Pb}$ from Ref. [28], and for ${ }^{209} \mathrm{Bi}$ from Ref. [12]. Note the differences in scale.

respective strength distributions have been deduced from only NRF resolved levels, too. Our aim is the identification and the quantification of the strongest photo-excited states of ${ }^{205} \mathrm{Tl}$ and an estimate of the corresponding strengths, whereas in other complementary NRF studies (see, e.g [8, 29]), in order to quantify the overall photo absorption cross section, the deduced strengths include the contribution of unresolved levels by an iterative deconvolution of the entire gamma-ray signal by means of statistical model. 
TABLE IV: Levels with possible branching (inelastic) transitions to lower-lying levels $E_{f}$. Given are the level excitation energies $E_{x}$, the transition energies $E_{\gamma}$ of both the elastic and inelastic transitions, the ground-state branching ratios $\frac{\Gamma_{0}}{\Gamma}$ under the assumption that all branchings have been observed in the present measurements, the ground-state widths $g \cdot \Gamma_{0}$ and the $B(E 1) \uparrow, B(M 1) \uparrow$ transition probabilities.

\begin{tabular}{|c|c|c|c|c|c|c|}
\hline $\begin{array}{c}E_{x} \\
(\mathrm{keV})\end{array}$ & $\begin{array}{c}E_{\gamma} \\
(\mathrm{keV})\end{array}$ & $\begin{array}{c}E_{f} \\
(\mathrm{keV})\end{array}$ & $\frac{\Gamma_{0}}{\Gamma_{a}}$ & $\begin{array}{c}g \Gamma_{0} \\
(\mathrm{eV})\end{array}$ & $\begin{array}{c}B(E 1) \\
\left(10^{-3} \mathrm{e}^{2} \mathrm{fm}^{2}\right)\end{array}$ & $\begin{array}{c}B(M 1) \\
\left(\mu^{2}\right)\end{array}$ \\
\hline $4938.2(2)$ & $\begin{array}{l}4938.2(2) \\
4731.6(7)\end{array}$ & $\begin{array}{c}0 \\
203.7\end{array}$ & $0.84(3)$ & $0.79(11)$ & $5.21(70)$ & $0.47(6)$ \\
\hline 4961.1(2) & $\begin{array}{c}4961.1(2) \\
4759.3(7)^{b} \\
4342.1(6)\end{array}$ & $\begin{array}{c}0 \\
203.7 \\
619.2\end{array}$ & $0.78(2)$ & $3.30(59)$ & $20.1(36)$ & $1.8(3)$ \\
\hline $4967.8(1)$ & $\begin{array}{c}4967.8(1) \\
4764.1(4)^{b} \\
4348.4(4)\end{array}$ & $\begin{array}{c}0 \\
203.7 \\
619.2\end{array}$ & $0.65(2)$ & $5.87(110)$ & $29.6(55)$ & $2.7(5)$ \\
\hline $5610.4(5)$ & $\begin{array}{l}5610.4(5) \\
5406.6(8)\end{array}$ & $\begin{array}{c}0 \\
203.7\end{array}$ & $0.73(2)$ & $1.89(39)$ & $7.5(15)$ & $0.68(14)$ \\
\hline $5686.2(3)$ & $\begin{array}{l}5686.2(3) \\
5480.2(5)\end{array}$ & $\begin{array}{c}0 \\
203.7\end{array}$ & $0.77(2)$ & $4.80(80)$ & $19.2(32)$ & $1.74(29)$ \\
\hline $5755.8(3)$ & $\begin{array}{l}5755.8(3) \\
5552.6(6)\end{array}$ & $\begin{array}{c}0 \\
203.7\end{array}$ & $0.78(7)$ & $4.66(71)$ & $18.1(23)$ & $1.64(21)$ \\
\hline $5781.4(6)$ & $\begin{array}{l}5781.4(6) \\
5577.1(7)\end{array}$ & $\begin{array}{c}0 \\
203.7\end{array}$ & $0.50(7)$ & $2.18(94)$ & $5.4(22)$ & $0.49(20)$ \\
\hline $5803.8(9)$ & $\begin{array}{l}5803.8(9) \\
5598.1(8)\end{array}$ & $\begin{array}{c}0 \\
203.7\end{array}$ & $0.61(7)$ & $1.80(59)$ & $5.4(17)$ & $0.49(15)$ \\
\hline $5963.8(18)$ & $\begin{array}{c}5963.8(18) \\
5343.6(9)\end{array}$ & $\begin{array}{c}0 \\
619.20\end{array}$ & $0.57(6)$ & $1.76(60)$ & $4.6(15)$ & $0.41(14)$ \\
\hline
\end{tabular}

${ }^{a}$ under the assumption of no other branching transitions
${ }^{b}$ confirmed as a branching transition in $\mathrm{HI} \gamma \mathrm{S}$ Experiment

A resonance-like structure was observed in $\mathrm{Tl}$ in early $(n, \gamma)$ experiments [13] as a bump at $\sim$ $5.5 \mathrm{MeV}$. NaI detectors used in that experiment did not allow for resolving its fine structure. A more detailed comparison of that data set to ours is, therefore, not possible.

\section{COMPARISON TO QPM PREDICTIONS}

The quasiparticle phonon model (QPM) [18] has been successfully applied in the past for describing the general behavior of low-lying dipole strength in the $A \approx 200$ mass region, e.g., for nuclei in the $\mathrm{Pb}$ chain (see, e.g., Refs. $[10,30]$ ). Thus, it has been used in the present work to calculate also the dipolestrength distribution of the odd-proton number $Z=$ 81 spherical nucleus ${ }^{205} \mathrm{Tl}$. In the following, we first 
provide a brief outline of the model in its application to spherical odd-mass nuclei. Afterwards, the QPM predictions will be compared to the experimentally deduced dipole-strength distribution. For a detailed review of the quasiparticle phonon model we refer to Refs. [31, 32].

\section{A. The QPM Formalism}

The ground and excited states of ${ }^{205} \mathrm{Tl}$ are described with a wave function which includes quasiparticle $\alpha_{j m}^{\dagger}$ (described on a mean field level), quasiparticle-phonon $\left[\alpha_{j^{\prime}}^{\dagger} Q_{\lambda i}^{\dagger}\right]_{j m}$, and quasiparticle two-phonon $\left[\left[\alpha_{j^{\prime}}^{\dagger} Q_{\lambda i}^{\dagger}\right]_{j^{\prime \prime}} Q_{\lambda^{\prime} i^{\prime}}^{\dagger}\right]_{j m}$ components (where $j m \equiv|n l j m\rangle)$ :

$$
\begin{gathered}
\Psi_{\nu}(j m)=C_{j}^{\nu}\left\{\alpha_{j m}^{\dagger}+\sum_{\lambda i j^{\prime}} D_{j^{\prime}}^{\lambda i}(j \nu)\left[\alpha_{j^{\prime}}^{\dagger} Q_{\lambda i}^{\dagger}\right]_{j m}+\right. \\
\left.\sum_{\lambda_{1} i_{1} \lambda_{2} i_{2} \lambda j^{\prime}} F_{j^{\prime} \lambda}^{\lambda_{1} i_{1} \lambda_{2} i_{2}}(J \nu)\left[\alpha_{j^{\prime}}^{\dagger}\left[Q_{\lambda_{1} i_{1}}^{\dagger} Q_{\lambda_{2} i_{2}}^{\dagger}\right]_{\lambda}\right]_{j m}\right\} \Psi_{0} .
\end{gathered}
$$

Here,

$$
\left[\alpha_{j^{\prime}}^{\dagger} Q_{\lambda i}^{\dagger}\right]_{j}=\sum_{m^{\prime} \mu} C_{j^{\prime} m^{\prime} \lambda \mu}^{j m} \alpha_{j^{\prime} m^{\prime}}^{\dagger} Q_{\lambda i \mu}^{\dagger}
$$

is an angular momentum coupling. The operator $Q_{\lambda^{\pi} i \mu}^{\dagger}$ is a phonon creation operator with the following quantum numbers: multipolarity $\lambda$, parity $\pi=$ \pm 1 , projection quantum number $\mu$, and the quasiparticle random phase approximation (QRPA) rootorder number $i$. It generates phonon excitations of a neighboring even-even core nucleus. The term $\Psi_{0}$ in Eq. (7) represents the quasiparticle/phonon vacuum, and the index $\nu=1,2, \ldots$ labels whether a state $j$ is the first, second, etc., state in the total energy spectrum of the system. Because of the spherical symmetry, all equations are degenerate with respect to the projection quantum numbers $m$ and $\mu$.

The spectrum of excited states $j$ and their wave functions, i.e., the coefficients $C, D$, and $F$ in Eq. (7), are obtained by diagonalization of the model Hamiltonian on a set of wave functions of the form of Eq. (7). The model Hamiltonian contains parts corresponding to the mean field for protons and neutrons (described by the Woods-Saxon potential), to the monopole proton-proton and neutron-neutron pairing, and to the residual interaction (in a separable form with the radial form factor given as a derivative of the mean field).

The spectrum of quasiparticles is obtained by solving the BCS equations with constant matrix elements of the monopole pairing. These equations also yield particle occupation numbers. The phonon spectrum of different multipolarities $\lambda^{\pi}$ is obtained from the QRPA equations. The strength of the residual interaction is fixed in the QPM on the QRPA levels by adjusting the collectivity of the lowest $2_{1}^{+}$and $3_{1}^{-}$states to their experimental values. The matrix elements of the interaction between different components of the wave function (7) are calculated microscopically without any free parameters:

$$
\begin{aligned}
\Gamma\left(j j^{\prime} \lambda i\right) & =\left\langle\alpha_{j}\left\|H_{Q P M}\right\|\left[\alpha_{j^{\prime}}^{\dagger} Q_{\lambda_{i}}^{\dagger}\right]_{j}\right\rangle \\
U_{\lambda_{2} i_{2}}^{\lambda_{1} i_{1}}(\lambda i) & =\left\langle Q_{\lambda i}\left\|H_{Q P M}\right\|\left[Q_{\lambda_{1} i_{1}}^{\dagger} Q_{\lambda_{2} i_{2}}^{\dagger}\right]\right\rangle .
\end{aligned}
$$

For the practical QPM calculations for ${ }^{205} \mathrm{Tl}$, we have used the same mean field and monopole pairing strength as in the case of the $\mathrm{Pb}$ isotopes [10]. The even-even nucleus ${ }^{204} \mathrm{Hg}$ has been chosen as the core having the same pairing in the proton system as ${ }^{205} \mathrm{Tl}$. In the wave function (7), natural parity phonons with $\lambda^{\pi}$ ranging from $1^{-}$to $7^{-}$and unnatural parity $1^{+}$phonons have been considered. All possible quasiparticle-phonon and quasiparticle two-phonon configurations with excitation energies below 6.5 and $7.5 \mathrm{MeV}$, respectively, have been included in the wave function (7).

Furthermore, the calculations have been restricted to states with spin and parity quantum numbers of $j^{\pi}=\frac{1}{2}^{ \pm}$and $j^{\pi}=\frac{3}{2}^{ \pm}$which can be excited from the $\frac{1}{2}^{+}$ground state of ${ }^{205} \mathrm{Tl}$ via electric and magnetic dipole transitions (which are mainly induced in NRF reactions). Such states are obtained by considering the coupling of the unpaired quasiparticle $\alpha_{j m}^{\dagger}$ from $3 s_{1 / 2}, 3 p_{1 / 2}, 3 p_{3 / 2}$ or $2 d_{3 / 2}$ orbitals of the configuration space to any one-phonon or two-phonon state of the even-even core which results in a $j^{\pi}=\frac{1}{2}^{ \pm}$or $\frac{3}{2}^{ \pm}$level.

\section{B. Comparison to Experimental Data}

The calculated strength distribution of ${ }^{205} \mathrm{Tl}$ is presented in Fig. 5 together with the results of the present NRF experiments. For the experimentally observed strength, E1 character is assumed in all cases. 


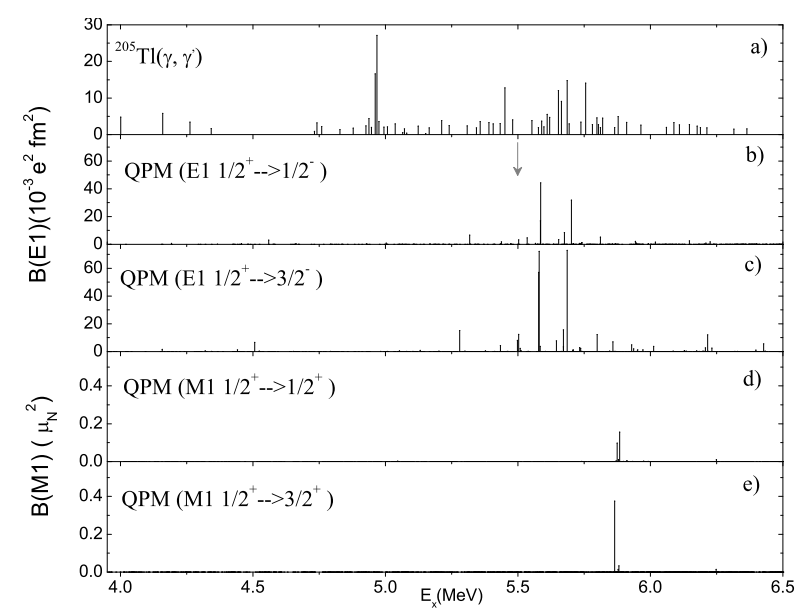

FIG. 5: Comparison of the observed reduced excitation probability distribution in ${ }^{205} \mathrm{Tl}$ (a) with results of QPM calculations (b,c,d,e) assuming a ${ }^{204} \mathrm{Hg}$ core. The gray arrow in panel (b) indicates the position of the strongest $1^{-}$phonon calculated for the even-even core ${ }^{204} \mathrm{Hg}$.

In the experiment, two groups of rather strong transitions have been observed; one around 4.9 MeV and one around 5.5 MeV excitation energy. Only one of these groups, namely the one at an energy of $5.5 \mathrm{MeV}$, is reproduced in the QPM calculation. However, this group is slightly shifted towards smaller excitation energy. Furthermore, the strength extracted from the QPM calculation is less fragmented in comparison to the experimental data. The fragmentation is underestimated in the calculations because $q p \otimes 3 p h$ components in the wave functions have been omitted due to their minor contribution to the total strength. The latter is about three times higher in comparison to the measured strength in the energy region between 5 and $6 \mathrm{MeV}$. However, a part of the strength may be missed in the experiment due to the limited experimental sensitivity, if it is strongly fragmented and distributed over many, only weakly excited states.

The ground state of ${ }^{205} \mathrm{Tl}\left(J^{\pi}=1 / 2^{+}\right)$is described via an almost pure quasiparticle state in the $3 s_{1 / 2}$ shell. The corresponding contribution of the $\alpha_{3 s_{1 / 2}}^{\dagger}$ quasiparticle to the ground-state wave function accounts to $97 \%$. A detailed look in the calculated wave functions of the excited states reveals that, although the number of the components contributing to the wave function given in Eq. (7) is of the order of a few thousand, in general, only a few of them carry noticeable dipole excitation strength. These

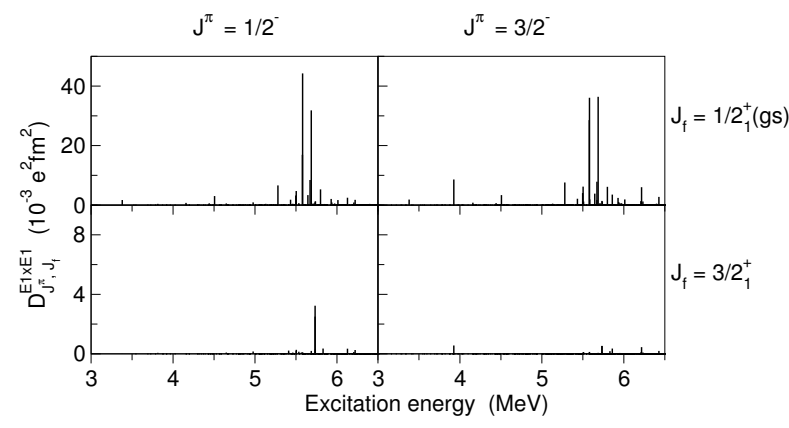

FIG. 6: The quantity $D_{J^{\pi}, J_{f}}^{E 1 \times{ }^{2}}$, Eq. (9), which describes g.s. $\rightarrow J^{\pi} \rightarrow J_{f}$ process.

are mainly single particle excitations to the $3 p_{1 / 2}$ shell and $q p \otimes 1 p h$ components. However, the contribution of single particle excitations to the wave functions are strongly suppressed to values of the sub-level due to the large energy gap between the $3 s_{1 / 2}$ and the $3 p_{3 / 2}$ or $3 p_{1 / 2} q p$ levels, respectively. The unpaired quasiparticle plays the role of a spectator. Therefore, the main part of the theoretical $E 1$ strength shown in Fig. 5 arises from $q p \otimes 1 p h$ components of the type $\left[\alpha_{3 s_{1 / 2}}^{\dagger} \otimes Q_{1-1}^{\dagger}\right]_{1 / 2^{-}\left(3 / 2^{-}\right)}$. Here, the strongest contribution is given by the lowest lying $1_{1}^{-}$one-phonon excitation of the core nucleus ${ }^{204} \mathrm{Hg}$ which is located at an excitation energy of $5.5 \mathrm{MeV}$ and carries a calculated transition strength of $B(E 1)=0.46 \mathrm{e}^{2} \mathrm{fm}^{2}$. Other $1^{-}$phonons of ${ }^{204} \mathrm{Hg}$ exhibit either only rather weak $B(E 1)$ transition strengths or are located above $7 \mathrm{MeV}$ excitation energy. They do not significantly contribute to the calculated strength distribution of ${ }^{205} \mathrm{Tl}$ below $6.5 \mathrm{MeV}$. Besides the $E 1$ excitations, only very weak $M 1$ strength has been found in the calculations around $5.8 \mathrm{MeV}$ which indicates that the strongest transitions observed in the experiment have $E 1$ character. The calculated $M 1$ strength is dominated by almost non-fragmented $\left[\alpha_{3 s_{1 / 2}}^{\dagger} \otimes Q_{1+4}^{\dagger}\right]_{1 / 2^{+}\left(3 / 2^{+}\right)}$configurations. In this case, the fourth $1^{+}$phonon of ${ }^{204} \mathrm{Hg}$ at an excitation energy of $5.82 \mathrm{MeV}$ has the strongest contribution to the $M 1$ strength distribution of ${ }^{205} \mathrm{Tl}$. This level corresponds to the wellknown isoscalar $1^{+}$level of ${ }^{208} \mathrm{~Pb}$ at $5.85 \mathrm{MeV}$ excitation energy. The lower-lying $1^{+}$phonons have significantly smaller $B(M 1)$ values.

In Fig. 6 we present the quantity:

$$
D_{J^{\pi}, J_{f}}^{E 1 \times E 1}=\sqrt{B\left(E 1, g . s . \rightarrow J^{\pi}\right) B\left(E 1, J^{\pi} \rightarrow J_{f}\right)}
$$


which mimics the process of the excitation of $J^{\pi}=$ $1 / 2^{-}$and $3 / 2^{-}$states from the ground state followed by the de-excitation to the ground state (top part) and to the first excited state $3 / 2^{+}$(bottom pannel) of ${ }^{205} \mathrm{Tl}$, respectively. The calculation predicts that the strongly excited states $1 / 2^{-}$and $3 / 2^{-}$ decay back to the ground state with almost $100 \%$ probability. This matches well with our experimental finding at $\mathrm{HI} \vec{\gamma} S$ facility of the measured groundstate branching ratios for the levels at $4961.1 \mathrm{keV}$ and $4967.8 \mathrm{keV}$ to the first excited state at 203.7 $\mathrm{keV}$ which amounts about $70 \%$.

\section{SUMMARY}

In this work, the dipole response of the oddproton nucleus ${ }^{205} \mathrm{Tl}$ has been investigated up to the neutron-separation threshold exploiting the method of nuclear resonance fluorescence. In total, $61 \gamma$ ray transitions in ${ }^{205} \mathrm{Tl}$ have been identified out of which 09 may be transitions to intermediate states. Furthermore, two transitions have been assigned to the second stable Thallium isotope, ${ }^{203} \mathrm{Tl}$. The extracted dipole-strength distribution of ${ }^{205} \mathrm{Tl}$ has been compared to neighboring nuclei in the $A \approx 200$ mass region.

QPM calculations considering a ${ }^{204} \mathrm{Hg}$ core, which exhibits a similar pairing, has been performed considering quasiparticle $\otimes \mathrm{N}$-phonon configurations $(\mathrm{N}=0,1,2)$. The calculation fails to reproduce the first group of observed strong transitions located at an excitation energy of $4.9 \mathrm{MeV}$, but reproduces the second group at $5.5 \mathrm{MeV}$ excitation en- ergy. The calculated distribution with a predominance of electric dipole character is shifted by 100 $\mathrm{keV}$ to lower energy with respect to the experimental results. The strongest transitions show a $3 s_{1 / 2} \rightarrow 3 s_{1 / 2} \otimes 1_{i}^{-}$structure, indicating that the unpaired quasi-particle behaves solely as a spectator.

The complementing measurement at $\mathrm{HI} \vec{\gamma} S$ emphasizes that, in order to distinguish decays via intermediate states from those directly to the ground state, measurements with mono-energetic photon beams, at least for the strongest excited states, are needed. Furthermore, to complete the systematics of low-lying dipole strength in the $N=126$ region, investigations on ${ }^{203} \mathrm{Tl}$ using an enriched target, and on stable $\mathrm{Hg}$ isotopes, which includes a long chain of odd and even mass nuclei, are highly desirable.

\section{ACKNOWLEDGEMENT}

The authors would like to thank the S-DALINAC and $\mathrm{HI} \gamma \mathrm{S}$ staff for the reliable operation of the facilities. We thank Dr. K. Hofman from the chemistry department at TU Darmstadt for her help during the $\mathrm{Tl}$ targets preparation. This work was supported by the Deutsche Forschungsgemeinschaft (Contract No. SFB 634), by the Alliance Program of the Helmholtz Association (HA216/EMMI) and by he U.S. Department of Energy, Office of Nuclear Physics under Grants No. DE-FG02-97ER41033, No. DE-FG02-97ER41041, and No. DE-FG0297ER41042.
[1] P. Lipas, Nucl. Phys. 82, 91 (1966), ISSN 0029-5582, URL http://www.sciencedirect.com/ science/article/pii/0029558266905256.

[2] W. Andrejtscheff, C. Kohstall, P. von Brentano, C. Fransen, U. Kneissl, N. Pietralla, and H. Pitz, Phys. Lett. B 506, 239 (2001), ISSN 0370-2693, URL http://www.sciencedirect.com/ science/article/pii/S0370269301003409.

[3] N. Pietralla, Phys. Rev. C 59, 2941 (1999), URL http://link.aps.org/doi/10.1103/PhysRevC. 59. 2941.

[4] D. Savran, T. Aumann, and A. Zilges, Prog. Part. Nucl. Phys. 70, 210 (2013), ISSN 0146-6410, URL http://www.sciencedirect.com/science/ article/pii/S0146641013000057.

[5] D. Bohle, A. Richter, W. Steffen, A. Dieperink,
N. L. Iudice, F. Palumbo, and O. Scholten, Phys. Lett. B 137, 27 (1984), ISSN 0370-2693, URL http://www.sciencedirect.com/science/ article/pii/0370269384910992.

[6] N. Pietralla, P. von Brentano, R.-D. Herzberg, U. Kneissl, N. Lo Iudice, H. Maser, H. H. Pitz, and A. Zilges, Phys. Rev. C 58, 184 (1998), URL http: //link.aps.org/doi/10.1103/PhysRevC.58.184.

[7] K. Heyde, P. von Neumann-Cosel, and A. Richter, Rev. Mod. Phys. 82, 2365 (2010).

[8] N. Benouaret, R. Schwengner, G. Rusev, F. Dönau, R. Beyer, M. Erhard, E. Grosse, A. R. Junghans, K. Kosev, C. Nair, et al., Phys. Rev. C 79, 014303 (2009), URL http://link.aps.org/doi/10.1103/ PhysRevC.79.014303.

[9] A. Makinaga, R. Schwengner, G. Rusev, F. Dönau, 
S. Frauendorf, D. Bemmerer, R. Beyer, P. Crespo, M. Erhard, A. R. Junghans, et al., Phys. Rev. C 82, 024314 (2010), URL http://link.aps.org/ doi/10.1103/PhysRevC.82.024314.

[10] J. Enders, P. von Brentano, J. Eberth, A. Fitzler, C. Fransen, R.-D. Herzberg, H. Kaiser, L. Käubler, P. von Neumann-Cosel, N. Pietralla, et al., Nucl. Phys. A 724, 243 (2003), ISSN 0375-9474, URL http://www.sciencedirect.com/ science/article/pii/S0375947403015549.

[11] N. Pietralla, T. Li, M. Fritzsche, M. Ahmed, T. Ahn, A. Costin, J. Enders, J. Li, S. Müller, P. von Neumann-Cosel, et al., Physics Letters B 681, 134 (2009), ISSN 0370-2693, URL http://www.sciencedirect.com/science/ article/pii/S0370269309011514.

[12] T. Chapuran, R. Vodhanel, and M. K. Brussel, Phys. Rev. C 22, 1420 (1980), URL http://link. aps.org/doi/10.1103/PhysRevC.22.1420.

[13] E. D. Earle, M. A. Lone, G. A. Bartholomew, W. J. McDonald, K. H. Bray, G. A. Moss, and G. C. Neilson, Can.J.Phys. 52, 989 (1974) 52, 989 (1974), ISSN 0375-9474, URL http://www . nrcresearchpress . com/doi/abs/10. 1139/p74-136. V83suTUpr6k.

[14] R. Laszewski and P. Axel, Phys. Rev. C 19, 342 (1979), URL http://dx.doi.org/10.1103/ PhysRevC.19.342.

[15] R. Laszewski, Phys. Rev. C 34, 342 (1986), URL http://dx.doi.org/10.1103/PhysRevC.34.1114.

[16] R. Barrett, K. Bray, B. Allen, and M. Kenny, Nucl. Phys. 278, 204 (1977), ISSN 0375-9474, URL http://www.sciencedirect.com/science/ article/pii/0375947486903805.

[17] M. Igashira, H. Kitazawa, M. Shimizu, H. Komano, and N. Yamamuro, Nucl. Phys. 457, 301 (1986), ISSN 0375-9474, URL http://www. sciencedirect. com/science/article/pii/0375947486903805.

[18] V. G. Soloviev, Theory of Atomic Nuclei, Quasiparticle and Phonons (Taylor \& Francis, 1992).

[19] U. Kneissl, H. Pitz, and A. Zilges, Prog. Part. Nucl. Phys. 37, 349 (1996), ISSN 0146-6410, URL http://www.sciencedirect.com/science/ article/pii/0146641096000555.

[20] K. Sonnabend, D. Savran, J. Beller, M. Büssing, A. Constantinescu, M. Elvers, J. Endres, M. Fritzsche, J. Glorius, J. Hasper, et al., Nucl. Instr. and Meth. A 640, 6 (2011), ISSN 0168-9002, URL http://www.sciencedirect.com/science/ article/pii/S0168900211005535.

[21] S. Agostinelli, J. Allison, K. Amako, J. Apostolakis, H. Araujo, P. Arce, M. Asai, D. Axen, S. Banerjee, G. Barrand, et al., Nucl. In- str. and Meth. A 506, 250 (2003), ISSN 0168-9002, URL http://www.sciencedirect.com/ science/article/pii/S0168900203013688.

[22] J. Endres, D. Savran, P. A. Butler, M. N. Harakeh, S. Harissopulos, R.-D. Herzberg, R. Krücken, A. Lagoyannis, E. Litvinova, N. Pietralla, et al., Phys. Rev. C 85, 064331 (2012), URL http:// link. aps.org/doi/10.1103/PhysRevC.85.064331.

[23] H. R. Weller, M. W. Ahmed, H. Gao, W. Tornow, Y. K. Wu, M. Gai, and R. Miskimen, Prog. Part. Nucl. Phys. 62, 257 (2009), ISSN 0146-6410, URL http://www.sciencedirect.com/science/ article/pii/S0146641008000434.

[24] A. Wolf, R. Moreh, A. Nof, O. Shahal, and J. Tenenbaum, Phys. Rev. C 6, 2276 (1972).

[25] M. Scheck, V. Y. Ponomarev, T. Aumann, J. Beller, M. Fritzsche, J. Isaak, J. H. Kelley, E. Kwan, N. Pietralla, R. Raut, et al., Phys. Rev. C 87, 051304 (2013), URL http://link.aps.org/doi/ 10.1103/PhysRevC.87.051304.

[26] C. Romig, J. Beller, J. Glorius, J. Isaak, J. H. Kelley, E. Kwan, N. Pietralla, V. Y. Ponomarev, A. Sauerwein, D. Savran, et al., Phys. Rev. C 88, 044331 (2013), URL http://link.aps.org/doi/10.1103/ PhysRevC.88.044331.

[27] C. Romig, D. Savran, J. Beller, J. Birkhan, A. Endres, M. Fritzsche, J. Glorius, J. Isaak, N. Pietralla, M. Scheck, et al., Physics Letters B 744, 369 (2015), ISSN 0370-2693, URL http://www.sciencedirect.com/science/ article/pii/S0370269315002567.

[28] R. Schwengner, R. Massarczyk, B. A. Brown, R. Beyer, F. Dönau, M. Erhard, E. Grosse, A. R. Junghans, K. Kosev, C. Nair, et al., Phys. Rev. C 81, 054315 (2010), URL http://link.aps.org/ doi/10.1103/PhysRevC.81.054315.

[29] A. Makinaga, R. Massarczyk, R. Schwengner, M. Beard, F. Dnau, M. Anders, D. Bemmerer, R. Beyer, R. Hannaske, A. R. Junghans, et al., Phys. Rev. C 90, 044301 (2014), URL http://link.aps. org/doi/10.1103/PhysRevC.82.024314.

[30] N. Ryezayeva, T. Hartmann, Y. Kalmykov, H. Lenske, P. von Neumann-Cosel, V. Yu. Ponomarev, A. Richter, A. Shevchenko, S. Volz, and J. Wambach, Phys. Rev. Lett. 89, 272502 (2002), URL http://link.aps.org/doi/10.1103/ PhysRevLett.89.272502.

[31] A. Vdovin, V. Voronov, V. Soloviev, and C. Stoyanov, Sov. J. Part. Nucl. 16 (1985).

[32] S. Gales, C. Stoyanov, and A. Vdovin, Phys. Rep.1988 166, 125 (1988). 